\title{
Les métaux marqueurs de la noyade
}

\author{
Metals and drowning
}

\section{Marc DEVEAUX*, Gilbert PÉPIN}

Laboratoire TOXLAB, 7 rue Jacques Cartier, 75018 Paris.

*Auteur à qui adresser la correspondance : Marc DEVEAUX, Laboratoire TOXLAB,

7, rue Jacques Cartier, F-75018 Paris.

Tél +33(0)1 58592800 - Fax +33(0)1 585928 01- E-mail : mdeveaux@labotoxlab.com

(Reçu le 30 mars 2007 ; accepté après modifications le 18 avril 2007)

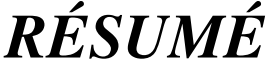

Le diagnostic médico-légal de noyade dans l'eau est rendu complexe du fait de trois mécanismes de décès possibles: noyade vraie, résultant de la pénétration de liquide dans les voies aériennes; hydrocution, sans pénétration hydrique; mort subite en situation immergée. Deux questions se posent habituellement : la mort est-elle survenue antérieurement à l'immersion? la noyade est-elle la cause de la mort? Les examens classiques macroscopiques et microscopiques peuvent révéler des lésions évocatrices de noyade, mais ces critères manquent de spécificité ; ceci a conduit à la description et à l'utilisation de marqueurs caractéristiques du passage de l'eau dans la circulation sanguine. Les variations des électrolytes (calcium, sodium, potassium, magnésium, chlore) ont été particulièrement suivies, mais sont difficiles à interpréter. C'est pourquoi deux métaux, le strontium ( $\mathrm{Sr}$ ) et le fer $(\mathrm{Fe})$, ont été étudiés durant la dernière décennie. Les évolutions récentes de l'ICP-MS représentent un apport intéressant pour ces dosages, qui peuvent être fait en routine. Nous présentons une méthode simple et rapide pour le dosage du Sr dans le sang et l'eau de noyade ou de découverte du cadavre. Les concentrations normales de Sr dans le sang varient de 10 à $50 \mu \mathrm{g} / \mathrm{L}$. Ce métal existe en quantités importantes, mais variables, dans les eaux douces. La concentration de Sr dans l'eau de mer est toujours très élevée. Lors d'une noyade par submersion, la pénétration d'eau dans le sang veineux pulmonaire se traduira par une hémodilution dans la petite circulation et une augmentation

\section{SUMMARY}

The medico-legal diagnosis of drowning is difficult because of three possible mechanisms of death : true drowning, due to water aspiration ; hydrocution, without water aspiration ; sudden death while immersion. Two questions are often asked: does death occurred before immersion ? is drowning the cause of death? Classical autopsy and microscopical findings could be helpful for the diagnosis of drowning, but these criterions are not totaly specific. Various thanatochemical parameters were studied (electrolytes and minerals including calcium, sodium, magnesium and chloride) in order to proof water aspiration. Unfortunately the interpretation of the result is difficult. Two metals, strontium $(\mathrm{Sr})$ and iron $(\mathrm{Fe})$ where also studied during the past ten years, and recent evolutions of ICP-MS appear to be very helpful for the simultaneous determination of these metals. We present here a fast and easy-to-use method for blood and water Sr determination. Noraml blood concentration of $\mathrm{Sr}$ ranged from 10 to $50 \mu \mathrm{g} / \mathrm{L}$. Strontium concentration is much more important in fresh water (at various concentrations) and is very high in sea water. In case of water aspiration, there is an hemodilution and Sr concentration in the left heart will rise with a significant difference. This value has to be compared with the Sr concentration in the water where the body was found. This can be used in both fresh and sea water. Iron concentrations measured in left and right heart are only useful for fresh water drowning. However, ICP-MS metals and metalloids determinations in blood and water are only a 
du Sr dans le sang du cour gauche. Il faut comparer ce résultat avec la concentration dans l'eau où a été retrouvé le cadavre. L'utilisation des concentrations sanguines en Fe reste limitée aux noyades en eau douce. On compare alors les teneurs en $\mathrm{Fe}$ du sang des cavités cardiaques droites et gauches : elle est plus importante à droite qu'à gauche. Quoiqu'il en soit, le dosage par ICP-MS de métaux et de métallö̈des dans le sang et l'eau de noyade ne peut être qu'un élément du faisceau d'arguments médico-légaux permettant de conclure à une noyade vitale.

\section{MOTS-CLÉS}

Métaux, strontium, sang, noyade.

\section{Introduction}

Le diagnostic médico-légal de la noyade dans l'eau est en général difficile. C'est l'existence de trois mécanismes possibles de décès qui le rend complexe : noyade vraie, par submersion, résultant de la respiration dans l'eau et donc de la pénétration de liquide dans les voies aériennes ; l'hydrocution, ou noyade par inhibition, sans pénétration hydrique; mort subite en situation immergée.

A part le cas évident où un témoin a observé qu'un individu vivant tombe dans l'eau et n'en est ressorti que mort, deux questions se posent quant aux circonstances du décès : la mort est-elle survenue antérieurement à l'immersion ? La noyade est-elle la cause de la mort? Les examens classiques macroscopiques et microscopiques peuvent révéler des lésions évocatrices de noyade, mais ces critères manquent de spécificité. Ceci a conduit à la description et à l'utilisation de marqueurs caractéristiques du passage de l'eau dans la circulation sanguine. Les variations des électrolytes ont été particulièrement suivies : calcium, sodium, potassium, chlore, mais sont difficiles à interpréter. C'est pourquoi, à partir des hypothèses d'Icard posées dès 1932 (1), deux métaux, le strontium $(\mathrm{Sr})$ et le fer $(\mathrm{Fe})$, ont été étudiés durant la dernière décennie en Europe (2-6). Ces métaux ont été dosés initialement par spectrométrie d'absorption atomique (SAA). Les évolutions récentes du plasma à couplage inductif relié à un spectromètre de masse (inductively coupled plasma-mass spectrometry, ICP-MS) représentent maintenant un apport intéressant pour ces dosages, qui peuvent être fait en routine, simultanément et rapidement. Nous présentons dans cet article l'apport du dosage de métaux dans le sang pour le diagnostic de noyade et décrivons une méthode de dosage simple et rapide du strontium par ICP-MS dans le sang et l'eau de découverte du cadavre. part of the combination of others findings, which appear to be a good compromise in arriving at a drowning conclusion.

Metals, strontium, blood, drowning.

\section{Critères diagnostiques positifs de la noyade $(7,8)$}

Les altérations cadavériques (hypertonie des globes oculaires, macération palmaire et plantaire, putréfaction d'évolution caractéristique) signent un séjour dans l'eau et permettent d'estimer grossièrement la durée de ce séjour, mais ce ne sont pas des signes de noyade. A l'examen du corps, les signes suivants sont notés : placards marbrés hémorragiques, cyanose de la face, écume mousseuse buccale et nasale : ils ne sont cependant pas toujours spécifiques. Pendant l'autopsie, on recherchera les signes d'asphyxie, la présence d'eau dans l'estomac, celle de sable et/ou de vase et/ou de boue dans les voies aériennes. Comme le décrit Durigon (8), c'est surtout l'aspect des poumons, distendus, ballonnés, lourds, avec un aspect d'emphysème hydro-aérique, qui confortera le diagnostic. Une congestion importante affecte l'ensemble des poumons et l'œdème alvéolaire est très important, peu chromophile.

\section{Marqueurs positifs de la noyade}

Les critères macroscopiques et microscopiques décrits précédemment manquent donc de spécificité. Des marqueurs positifs, plus caractéristiques, ont été décrits. On distingue la mise en évidence de l'hémodilution ou de l'hémoconcentration et la mise en évidence de la pénétration d'éléments extérieurs (marqueurs extrinsèques).

\section{Mise en évidence de l'hémodilution}

Le passage d'eau douce dans le sang veineux pulmonaire entraine une hémodilution importante dans la petite circulation. Il faudra donc au début de l'autopsie prélever du sang dans le cœur droitet dans le cœurgauche eteffectuer 
des comparaisons entre les deux prélèvements. Durigon (8) a passé en revue les éléments à étudier : mesure de la densité ; étude de la variation globale de la concentration en électrolytes par la méthode cryoscopique; études des variations individuelles des électrolytes (sodium, potassium, chlore, calcium, magnésium par exemple) ; dosage de l'hémoglobine, des protéines. Les méthodes de dosage sont celles classiquement utilisées en biochimie. Tous ces paramètres sont utilisés avec plus ou moins de succès, mais jamais seuls car aucun n'est vraiment sélectif : leur étude ne fait qu'alimenter un faisceau d'arguments permettant d'arriver au diagnostic de noyade vitale (913). Il serait ainsi possible de différencier une noyade en eau douce d'une noyade en eau de mer: dans le premier cas, le sodium, le potassium, le magnésium et le chlore sont diminués, alors que le calcium est augmenté.

De rares travaux $(6,14)$ ont mis l'accent sur l'utilisation de la mesure comparative du fer sanguin : la concentration en fer est plus importante dans le cœur droit (FeCD) que dans le cœur gauche (FeCG). Le fer peut être dosé par colorimétrie, la SAA ou l'ICP-MS n'étant absolument pas impératives, car le dosage sanguin du fer est réalisé en routine dans de nombreux laboratoires de biochimie courante. Sur 26 cas étudiés par Lorin (6), la différence $\mathrm{FeCD}-\mathrm{FeCG}$ est en moyenne de 67,7 +/- 86,2 $\mu \mathrm{mol} / \mathrm{L}$, avec une grande étendue de 12 à $387 \mu \mathrm{mol} / \mathrm{L}$, due vraisemblablement à des agonies de durées différentes. Ce marqueur de l'hémodilution est de bonne présomption si le corps n'a pas séjourné trop longtemps dans l'eau. Il est donc bien discriminatif, mais il est cependant limité aux noyades en eau douce (6). Pour des problèmes techniques, la présence de caillot ou le sang putréfié implique l'utilisation de l'ICP-MS.

\section{Mise en évidence de l'hémoconcentration}

Lors d'une noyade en eau de mer, le passage de cette eau hypertonique dans le sang veineux pulmonaire provoque une hémoconcentration. Des travaux initiés par Piette (15) et poursuivis par Azparren $(5,16,17)$ ont montré que l'on pouvait utiliser les différences de concentration du strontium entre le sang du cœur gauche ( $\mathrm{SrCG}$ ) et celui du cœur droit ( $\mathrm{SrCD})$. Les auteurs concluent que si $\mathrm{SrCG}$ est supérieur à $\mathrm{SrCD}$, on dispose d'un argument supplémentaire pour le diagnostic de noyade vitale. Ce paramètre n'est utilisable que pour une noyade en eau de mer. Il est préférable que le dosage soit effectué par ICP-MS, pour s'affranchir des problèmes de caillots et de putréfaction. Cependant tous les résultats des études d'Azparren précédemment cités ont été obtenus par SAA électrothermique avec correction par effet Zeeman.

\section{Mise en évidence de la pénétration d'un marqueur extérieur}

\section{Éléments du plancton}

Les diatomées sont des algues unicellulaires microscopiques à coque siliceuse. Leur recherche est difficile et nécessite un protocole très rigoureux. Elle s'effectue par rapport à un échantillon d'eau comparatif (18)et doit aller jusqu'àl'identification et la quantification. Cette recherche s'effectue dans les poumons, le sang et la moelle osseuse.

D'autres éléments du plancton (protozoaires, germes de la flore aquatique) peuvent être recherchés dans le sang. Comme le note Durigon (8), cette méthode est inutilisable dès le début de la putréfaction ou si l'eau ne contient pas de plancton, comme c'est le cas pour les eaux de baignoires par exemple.

\section{Dosage du strontium}

La concentration de strontium dans l'eau douce (SrED) et dans l'eau de mer (SrEM) étant beaucoup plus élevée que dans le sang $(\mathrm{SrSg})$, Icard (1) avait émis l'hypothèse qu'après une noyade vitale, la concentration de strontium pouvait augmenter dans le sang. Cette augmentation devait être en rapport avec SrED ou SrEM. L'idée était donc née de doser le strontium dans le sang et dans l'eau de la noyade (ou à défaut l'eau du lieu de découverte du cadavre) et de comparer ces valeurs entre elles. On pouvait donc s'affranchir de la différence $\mathrm{SrD}-\mathrm{SrG}$, car les différences de concentrations entre l'eau et le sang sont la plupart du temps considérables. Le tableau I donne une liste de valeurs de SrED et de SrEM retrouvées dans la littérature. Comme elles sont éparses et finalement assez rares, nous établissons actuellement une base de données de concentrations de strontium dans les eaux de ville, rivières, lacs, étangs, canaux et bords de mer.

De nombreux auteurs, essentiellement européens, se sont attachés à préciser la valeur diagnostique de ce test $(2-5,19)$, sachant que les premières études avaient été effectuées sur des lapins (19).

Il ne semble pas qu' un consensus se soit fait sur cette valeur prédictive, mais la comparaison des concentrations $\mathrm{SrSg}$ SrED ou SrSg-SrEM reste un élément indispensable du faisceau d'arguments permettant d'affirmer la noyade par asphyxie. Les valeurs de $\mathrm{SrSg}$ actuellement considérées comme normales dans le sang de cadavre vont de 1 à $43 \mu \mathrm{g} / \mathrm{L}(3,5,15,20,21)$. Les concentrations établies comme physiologiques dans le sang total vont de 9 à $41 \mu \mathrm{g} / \mathrm{L}(20)$ et vont jusqu'à $80 \mu \mathrm{g} / \mathrm{L}$ dans le sérum. Ce chiffre peut atteindre $9600 \mu \mathrm{g} / \mathrm{L}$ chez des patientes ostéoporotiques recevant du ranélate de strontium (Protelos ${ }^{\circledR}$, Laboratoire Servier Médical) à la dose quotidienne de $2 \mathrm{~g} / \mathrm{j}$ (Chanu, communication personnelle, 2007). 
Tableau I : Teneurs moyennes en strontium dans différentes eaux (en $\mu g / L)$. Légende $: \S$ : observations personnelles ; $B:$ Belgique. $S P$ : Espagne ; IdF : Ile de France

\begin{tabular}{|c|c|c|}
\hline Nature de l'eau & & (ref). \\
\hline \multicolumn{3}{|l|}{ Eau douce (cours d'eau) } \\
\hline Isère & 860 & (2) \\
\hline Seine & $200-500$ & $(4, \S)$ \\
\hline Aube & 360 & $(\S)$ \\
\hline Marne & 326 & $(\S)$ \\
\hline Leie (B) & 450 & (15) \\
\hline Limoges & $<50$ & $(\S)$ \\
\hline Martinique & 75 & $(\S)$ \\
\hline Région minière (SP) & $>1000$ & $(16)$ \\
\hline \multicolumn{3}{|l|}{ Eau douce stagnante } \\
\hline Créteil & 4120 & $(\S)$ \\
\hline Polders (B) & 1100 & (15) \\
\hline Canaux (IdF) & $800-1200$ & $(4, \S)$ \\
\hline Etang (IdF) & $50-400$ & (4) \\
\hline $\begin{array}{l}\text { Eaux stagnantes } \\
\text { après orage }\end{array}$ & $<50$ & $(\S)$ \\
\hline \multicolumn{3}{|l|}{ Eau du robinet } \\
\hline Grenoble & 680 & (2) \\
\hline Strasbourg & 600 & $(22)$ \\
\hline Belgique & 300 & (15) \\
\hline Paris & 243 & (4) \\
\hline \multicolumn{3}{|l|}{ Piscine } \\
\hline Paris & 1384 & $(\S)$ \\
\hline \multicolumn{3}{|l|}{ Eau de mer } \\
\hline Mer du Nord & $6000-9000$ & $(15, \S)$ \\
\hline Manche (port) & 5000 & (20) \\
\hline Méditerranée & 29000 & $(\S)$ \\
\hline Atlantique (SP) & $3000-9000$ & $(5,17)$ \\
\hline
\end{tabular}

\section{Méthodes de dosage du strontium}

Le dosage du strontium dans le sang de noyés a été effectué par SAA en mode flamme (19), par SAA électrothermique $(3,4,21)$, par SAA électrothermique avec correction par effet Zeeman $(5,16)$. L'utilisation de la spectrométrie d'émission par plasma est apparue avec les travaux de Piette (15) avec un système optique (ICP-AES). La minéralisation du prélèvement sanguin en milieu acide était effectuée par micro-ondes.

Des travaux plus récents ont établi l'état de l'art sur le dosage de différents métaux, dont le strontium, dans les milieux biologiques par ICP-MS, avec des méthodes validés $(20,23,24)$, sur des sujets vivants et dans des cas médico-légaux.

Dans les cas de noyade avérée ou suspectée, nous dosons habituellement le strontium dans le sang cardiaque et, lorsque nous en disposons, dans l'eau de noyade. L'appareil utilisé est un ICP-MS XSeries II (Thermo Fisher Scientific, Courtabœuf). Il est équipé d'un nébuliseur concentrique associé à une chambre de nébulisation simple passage à bille d'impact, refroidie à $+3^{\circ} \mathrm{C}$ par effet Peltier. Les paramètres instrumentaux sont les suivants : puissance de la torche $1380 \mathrm{~W}$; pression d'argon au niveau du nébuliseur : 2,0 à 2,2 bars; interface : cônes échantillonneurs et écorceurs de $1 \mathrm{~mm}$ et $0,7 \mathrm{~mm}$ respectivement ; vide au niveau du quadripôle : 1,0 à $3,0.10^{-7}$ mbar ; pompe péristaltique : $15 \mathrm{rpm}$.

Les dosages dans le sang et l'eau de noyade sont effectués après simple dilution, respectivement au 1/40 et au $1 / 100$ dans de l'acide nitrique à $0,65 \%(\mathrm{p} / \mathrm{v})$. L'étalon interne est le rhodium $(2 \mathrm{~mL}$ d'une solution à $10 \mu \mathrm{g} / \mathrm{L})$. La méthode a été validée dans des solutions aqueuses, avec vérification par la méthode des ajouts dosés $(25,26)$. Elle est linéaire de 0,02 à $200 \mu \mathrm{g} / \mathrm{L}$, la limite de quantification est de $0,02 \mu \mathrm{g} / \mathrm{L}$, la limite de détection de $0,01 \mathrm{~g} / \mathrm{L}$. La répétabilité et la fidélité intermédiaire sont excellentes (respectivement $0,4 \%$ et $1 \%$ à $10 \mu \mathrm{g} / \mathrm{L}$ et $100 \mu \mathrm{g} / \mathrm{L})$.

\section{Discussion}

Azparren estime que l'interprétation des concentrations de $\mathrm{Sr}$ est simplifiée si $\mathrm{SrSg}$ est supérieure à $160 \mu \mathrm{g} / \mathrm{L}$ (27) tout en restant inférieure ou du même ordre de grandeur que SrED (ou SrEM) : on a là un argument supplémentaire en faveur d'une noyade vitale. La différence SrCG - SrCD ne serait utilisable pour une noyade vitale en eau douce que si on peut la rapporter à SrED, étant donné la grande variabilité (selon le lieu et la saison) de la teneur en $\mathrm{Sr}$ des eaux douces ; Azparren propose un arbre de décision assez simple en fonction de $\operatorname{SrED}(28,29)$. Aucun autre travail publié à ce jour n'a permis de vérifier ces hypothèses.

Si les différences entre les valeurs mesurées sont moins importantes, ou encore après un séjour dans l'eau de plus de 2 à 3 semaines, ou si des blessures ont favorisé l'entrée de l'eau dans le corps (avec donc pénétration d'eau dans les organes et le sang), l'interprétation des résultats devient très délicate. De même, on sait que la durée de l'agonie fait augmenter la concentration de Sr dans le sang cardiaque (20). Enfin, dans les cas de noyade-suicides dans des baignoires, où la victime a souvent absorbé beaucoup d'alcool et de tranquillisants ou hypnotiques, la mort survient rapidement, sans que le sujet inhale beaucoup d'eau : $\mathrm{SrSg}$ sera donc peu élevé (7)

En cas de noyade en eau de mer, une nouvelle piste de travail vient d'être évoquée par Goullé (30), reprenant une hypothèse émise par Powitz (31) : il s'agit de la mesure du brome (Br) sanguin, comparée à la concentration de $\mathrm{Br}$ retrouvée dans l'eau de noyade. La teneur en $\mathrm{Br}$ de l'eau de mer est 4 à 6 fois supérieure à la teneur en strontium (mais seulement 2 fois pour des eaux de rivière), ce qui en ferait un paramètre supplémentaire tout à fait utilisable $(30,32,33)$. Les résultats préliminaires montrent que les interférences peuvent être nombreuses, mais ils semblent prometteurs. 


\section{Conclusion}

Même si l'autopsie révèle des lésions évocatrices de noyade et que la recherche des diatomées reste utile, le dosage simultané du strontium dans le sang et dans l'eau de découverte du corps reste absolument indispensable : c'est un élément supplémentaire du faisceau de preuves médico-légales permettant de conclure à une noyade vitale. Les éléments essentiels utilisables dans le cas de découverte de cadavre en eau douce peuvent être résumés comme suit :

- valeurs normales du Sr dans le sang : 10-50 $\mu \mathrm{g} / \mathrm{L}$

- Sr dans le sang après noyade vitale : 70-500 $\mu \mathrm{g} / \mathrm{L}$

- Sr dans le sang en cas d'immersion après la mort : $15-80 \mu \mathrm{g} / \mathrm{L}$

Le dosage simultané d'autres métaux et métalloïdes (fer, brome) pourrait augmenter la spécificité de ce test et l'argument du surcoût opposé par Piette (34) n'est plus recevable aujourd'hui du fait de la démocratisation de l'ICP-MS.

\section{Remerciements}

Les auteurs remercient M. Francis Billaut et M. Florent Brousse pour leur aide technique dans la réalisation des dosages de strontium.

\section{Références}

1. Icard S. La preuve de la mort par submersion suivant qu'elle a lieu dans une rivière ou dans la mer. Rev. Pathol. Comparée Hyg. Générale. 1932 ; 32 : 559-71.

2. Barret L., Stoklov M., Meyran S., Danel V., Marka Ch., Faure J. Intérêt du dosage du strontium sanguin comme marquer de la noyade vitale. J. Méd. Lég. Droit Méd. 1987 ; 30(1) : 21-6.

3. Piette M., Timperman J., Parisis N. Serum strontium estimation as a medico-legal diagnotic indicator of drowning. Med. Sci. Law. 1989 ; 29(2) : 162-71.

4. Fornes P., Pépin G., Heudes D., Lecomte D. Diagnosis of drowning by combined computer-assisted histomorphometry of lungs with blood strontium determination. J. Forensic. Sci. 1998 ; 43(4) : 772-6.

5. Azparren J.E., de la Rosa I., Sancho M. Biventricular measurement of blood strontium in real cases of drowning. Forensic Sci. Int. 1994 ; 69 : 139-48.

6. Lorin de la Granmaison G., Leterreux M., Lasseuguette K., Alvarez J.C., de Mazancourt Ph., Durigon M. Study of the diagnostic value of iron in fresh water drowning. Forensic Sci. Int. 2006 ; 157 : 117-20.

7. Campana J.P., Fornes P. Asphyxies mécaniques. In : Campana J.P. (coordinateur) Principes de Médecine Légale ; Rueil : Arnette, 2003 ; 105-23.

8. Durigon M. Diagnostic d'une noyade. Méd. Lég. Hosp. $2000 ; 3(2): 59-60$.

9. Moritz A.R. Chemical methods for the determination of death by drowning. Physiol. Rev. 1944 ; 24 : 70-88.
10. Yagil Y., Stalnikowicz R., Michaeli J., Mogle P. Near drowning in the Dead Sea. Electrolyte imbalances and therapeutic implications. Arch. Int. Med. 1985 ; 145(1) : 50-3.

11. Zhu B.L., Ishikawa T., Quan L. et al. Evaluation of postmortem serum calcium and magnesium levels in relation to the causes of death in forensic autopsy. Forensic Sci. Int. 2005 ; 155 : 18-23.

12. Inoue H., Ishida T., Tsuji A., Kudo K., Ikeda N. Electrolyte analysis in pleural effusion as an indicator of the drowning medium. Leg. Med. (Tokyo). $2005 ; 7(2)$ : 96-102.

13. Coutselinis A., Boukis D. The estimation of $\mathrm{Mg}$ concenation in cerebrospinal fluid as a method of drowning diagnosis in sea water. Forensic Sci. 1976 ; 7 : 109-11.

14. Gettler O.A. A method for determination of death by drowning. JAMA. $1921 ; 66: 1650$.

15. Piette M., Desmet B., Dams R. Determination of strontium in human blood by ICP-AES. Sci. Total Environ. 1994 ; 141(1-3) : 269-73.

16. Azparren J.E., Vallejo G., Reyes E., Herranz A., Sancho M. Study of the diagnostic value of strontium chloride, haemoglobin and diatoms in immersion cases. Forensic Sci. Int. 1998 ; 91 : 123-32.

17. Azparren J.E., Ortega A., Bueno H., Andreu M. Blood strontium concentration related to the length of the agonal period in seawater drowning cases. Forensic Sci. Int. $2000 ; 108: 51-60$.

18. Ludes B., Coste M., North N., Doray S., Tracqui A., Kintz P. Diatom analysis in victim's tissues as an indicator of the site of drowning. Int. J. Legal Med. 1999 ; 112 : 163-6.

19. Abdallah A.M., Hassan S.A., Kabil M.A., Ghanim A.E.E. Serum strontium estimation as a diagnostic criterion of the type of drowning water. Forensic Sci. Int. 1985 ; 28(1) : 47-52.

20. Goullé J.P., Mahieu L., Maignant V., Bouige D., Saussereau E., Lacroix C. Valeurs usuelles des métaux et métalloïdes dans le sang total et les urines par ICP-MS chez cinquante quatre sujets décédés. Ann. Toxicol. Anal. 2007 ; 17(1) : 43-51.

21. Goullé J.P., Mahieu L., Castermant J. et al. Metal and metalloid multi-elementary ICP-MS validation in whole blood, plasma, urine and hair. Reference values. Forensic Sci. Int. $2005 ; 153: 39-44$.

22. Tracqui A., Ludes B., Bosque M.A., Mangin P. Diagnosis of drowning by blood strontium determination versus diatom test. In : Collery $\mathrm{Ph}$. et al., eds. Metal ions in biology and medicine ; vol 4. Paris : John Libbey Eurotext, 1996 ; 499-501.

23. Labat L., Dehon B., Dhorne C., Lhermitte M. Dosage de métaux par ICP-MS dans différents milieux biologiques. Ann. Toxicol. Anal. 2003 ; 15(4) : 281-6.

24. Goullé J.P., Mahieu L., Neveu N. et coll. Dosage multiélémentaire des métaux et métalloïdes dans les milieux biologiques par ICP-MS : valeurs usuelles chez 100 témoins. Ann. Toxicol. Anal. 2004 ; 16(4) : 261-8.

25. Goullé J.P., Mahieu L., Castermant J. et coll. Validation d'une technique de dosage multi-élémentaire des métaux par ICP-MS dans les milieux biologiques. Ann. Toxicol. Anal. 2003 ; 15(4) : 271-80. 
26. Gruson A., Groupe de travail «Accréditation » de la SFTA. Aide à la validation des méthodes en toxicologie et suivi thérapeutique pharmacologique. $2005 ; 17(3$, suppl 1) : 1-20.

27. Azparren J.E., Fernandez-Rodriguez A., Vallejo G. Diagnosing death by drowning in fresh water using blood strontium as an indicator. Forensic Sci. Int. 2003 ; 137 : $55-9$.

28. Azparren J.E., Cubero C., Perucha E., Martinez P., Vallejo G. Comparison between lung weight and blood strontium found in seawater. Forensic Sci. Int. (2006), doi : 10.1016/j.forsciint.2006.07.001.

29. Azparren J.E., Perucha E., Martinez P., Munoz R., Vallejo G. Factors affecting strontium absorption in drownings. Forensic Sci. Int. (2006), doi:10.1016/ j.forsciint.2006.07.003.

30. Goullé J.P., Mahieu L., Maignant V., Bouige D., Saussereau E., Lacroix C. Métaux et métalloides : valeurs usuelles dans le sang et les urines chez 50 sujets décédés. Ann. Toxicol. Anal. 2006 ; 18(3) : 160-1.

31. Powitz G. Determination de l'incorporation du brome par des cadavres ayant séjournés en eau de mer [en allemand]. Beitr. Gerichtl. Med. 1992 ; 50 : 117-9.

32. Swenson H. Why is the ocean salty ? http://www.palomar. edu/oceanography/salty_ocean.htm Site consulté en décembre 2006.

33. Scott M.R. Ocean chemical processes. http://www. waterencyclopedia.com/Mi-Oc/Ocean-ChemicalProcesses.html Site consulté en décembre 2006

34. Piette M.H.A., De Letter E.A. Drowning : still a difficult autopsy diagnosis. Forensic Sci. Inter. 2006 ; 163(1-2) : $1-9$. 